\title{
Layer-by-layer assembled graphene oxide films and barrier properties of thermally reduced graphene oxide membranes
}

\author{
Seon-Guk Kim ${ }^{1,2}$, Ok-Kyung Park ${ }^{1,2}$, Joong Hee Lee ${ }^{2, \star}$ and Bon-Cheol Ku, ${ }^{1, \star}$ \\ ${ }^{1}$ Carbon Convergence Materials Research Center, Institute of Advanced Composites Materials, Korea Institute of Science and Technology, \\ Wanju 565-905, Korea \\ ${ }^{2}$ Department of BIN Fusion Technology and Department of Polymer and Nano Science and Technology, Chonbuk National University, \\ Jeonju, 561-759, Korea
}

\author{
Article Info \\ Received 15 August 2013 \\ Accepted 5 October 2013 \\ *Corresponding Author \\ E-mail: cnt@kist.re.kr \\ jhl@jbnu.ac.kr \\ Tel: $+82-63-219-8132$

\section{Open Access} \\ DOI: http://dx.doi.org/ \\ 10.5714/CL.2013.14.4.247 \\ This is an Open Access article distributed \\ under the terms of the Creative Commons \\ Attribution Non-Commercial License \\ (http://creativecommons.org/licenses/ \\ by-nc/3.0/) which permits unrestricted \\ non-commercial use, distribution, and \\ reproduction in any medium, provided \\ the original work is properly cited.
}

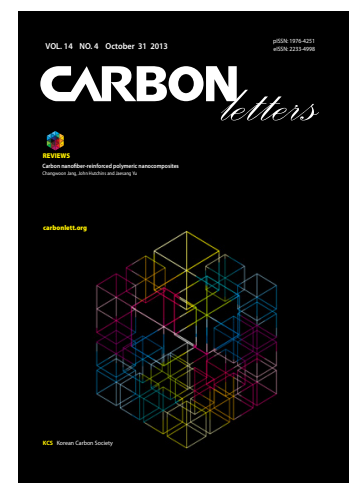

http://carbonlett.org

pISSN: $1976-4251$

elSSN: 2233-4998

Copyright $\odot$ Korean Carbon Society

\begin{abstract}
In this study, we present a facile method of fabricating graphene oxide (GO) films on the surface of polyimide (PI) via layer-by-layer (LBL) assembly of charged GO. The positively charged amino-phenyl functionalized GO (APGO) is alternatively complexed with the negatively charged GO through an electrostatic LBL assembly process. Furthermore, we investigated the water vapor transmission rate and oxygen transmission rate of the prepared (reduced $\mathrm{GO}[\mathrm{rGO}] / \mathrm{rAPGO})_{10}$ deposited PI film (rGO/rAPGO/PI) and pure PI film. The water vapor transmission rate of the GO and APGO-coated PI composite film was increased due to the intrinsically hydrophilic property of the charged composite films. However, the oxygen transmission rate was decreased from 220 to $78 \mathrm{~cm}^{3} / \mathrm{m}^{2}$ day atm, due to the barrier effect of the graphene films on the PI surface. Since the proposed method allows for large-scale production of graphene films, it is considered to have potential for utilization in various applications.
\end{abstract}

Key words: layer-by-layer assembly, graphene oxide, water vapor transmission rate, oxygen transmission rate

\section{Introduction}

Graphene is a 2-dimensional carbon material discovered by Professor Andre Geim et al. [1]. It consists of a hexagonal array of $s p^{2}$-bonded carbon atoms. The unique properties of graphene provide potential uses for a wide range of applications including nano-electronics, super-capacitors, hydrogen storage and nanocomposite [1-4].

High-performance polymer composites can be fabricated by incorporating nano-fillers into polymer matrices $[5,6]$. Generally, carbon nanotubes (CNTs) have been used to fabricate high-performance polymer composites [7]. However, graphene has already been experimentally shown to exhibit superior gas barrier and mechanical properties compared to CNTs [8].

The incorporation of graphene can reduce gas permeation through composite membranes by maximizing the gas-diffusion path-length [9]. Various methods, such as Langmuir-Blodgtt film assembly, vacuum filtration and air-water interface collection, have been proposed for the fabrication of graphene films. However, the uniformity of the graphene films prepared by the methods mentioned above is inadequate to fabricate high barrier membranes. This is because of the incompletely interconnected networks between thin layer graphene sheets. The layer-by-layer (LBL) assembly method can be considered as a promising candidate for generating uniform graphene films.

LBL assembly, known as a nanofabrication technique most commonly using oppositely charged polyelectrolytes, has been used to prepare a variety of multifunctional thin films due to its simplicity, robustness and versatility $[10,11]$. LBL assembled films can be prepared by depositing alternately-charged polymer and nanoparticles onto a substrate with controlled 


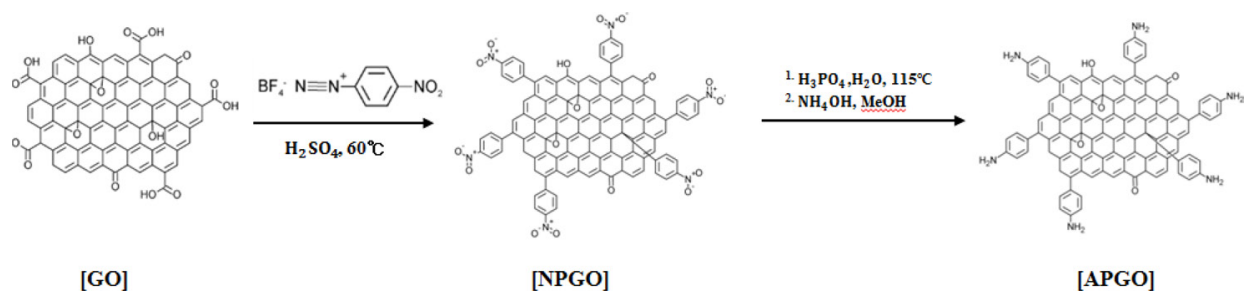

Fig. 1. Schematic representation of amino-phenyl functionalized graphene oxide (APGO). NPGO: nitro-phenyl functionalized GO.

thickness via electrostatic attraction or hydrogen bonding. This precisely controlled deposition of ingredients has already been used to generate superior gas barrier films. LBL film properties providing high transparency and gas barrier properties can be precisely controlled by tailoring concentration, temperature, and $\mathrm{pH}$ of the polyelectrolyte solution [12-15].

Herein, we have presented a facile approach for preparing graphene composite films on polyimide (PI) membranes with effective gas barrier properties. Positively charged amino-phenyl functionalized grapheme oxide (APGO) is alternatively complexed with the negatively charged GO through an LBL assembly process. During this process, imidization can convert GO film to graphene film.

\section{Experimental}

\subsection{Materials}

Natural Graphite powder (microcrystal grade, APS 2-15 $\mu$ m, 99.9995\% purity, metals basis) was purchased from Alfa Aesar. Nitrobenzene diazonium-tetrafluoroborates, phosphoric acid and ammonium hydroxide were purchased from SigmaAldrich (USA). The polyamic acid (PAA) was prepared via in situ polymerization. Pyromellitic dianhydride was purchased from Lonza (China); the 4, 4-oxidianiline, and 1-methyl-2- pyrrolidinone from Sigma-Aldrich (USA); and the 1,4-Diazabicyclo[2,2,2]octane from TCI (Japan). All chemicals were used as received; without further purification.

\subsection{Preparation of APGO}

The oxidation of graphite was carried out following a modified Hummer's method [16]. $\mathrm{GO}(0.1 \mathrm{~g})$ was immersed in $\mathrm{H}_{2} \mathrm{SO}_{4}$ $(100 \mathrm{~mL})$ and sonicated for $30 \mathrm{~min}$; followed by the addition of $1.8 \mathrm{~g}$ of nitrobenzene diazonium-tetrafluoroborate. The mixture was magnetically stirred vigorously at $60^{\circ} \mathrm{C}$ for $1 \mathrm{~h}$. After cooling to room temperature, the mixture was diluted and washed with dimethylformamide. Finally, the product was washed with ethanol and dried at $60^{\circ} \mathrm{C}$ for $24 \mathrm{~h}$ in a vacuum oven to get the nitro-phenyl functionalized GO (NPGO). Then, $0.1 \mathrm{~g}$ of dried NPGO was added to a solution of phosphoric acid (45 g) and DI-water $(50 \mathrm{~mL})$. The mixture was stirred at $115^{\circ} \mathrm{C}$ for $6 \mathrm{~h}$. After cooling to room temperature, the reactant mixture was transferred into a mixed solution of $200 \mathrm{~mL}$ of ammonium hydroxide and $100 \mathrm{~mL}$ of methanol. Next, the mixture was diluted with DI-water and stirred for $6 \mathrm{~h}$ for desalination. The APGO was washed with ethanol and dried at $60^{\circ} \mathrm{C}$ for $24 \mathrm{~h}$ in a vacuum oven (Fig. 1).

\subsection{LBL assembly film-fabrication}

Negatively charged GO and positively charged APGO solution were prepared using $18.2 \mathrm{M} \Omega$ DI-water at the concentration of $0.5 \mathrm{mg} / \mathrm{mL}$. The $\mathrm{pH}$ value of each solution was maintained at approximately 10 using $\mathrm{NaOH}$. Positively charged APGO solution was dropped on the PAA substrate the amount of water droplets sprayed through the spray gun, and then spin-coated at $3000 \mathrm{rpm}$ for $40 \mathrm{~s}$; followed by drying at $80^{\circ} \mathrm{C}$. Negatively charged GO was deposited using the same methods. Ten deposition cycles of GO and AGPGO bi-layers were prepared on PAA films; followed by the buildup sequence of GO and APGO. The LBL-assembled reduced $\mathrm{GO}(\mathrm{rGO}) / \mathrm{rAPGO} / \mathrm{PI}$ film was subjected to thermal curing of the PAA in an oven with steps of $100^{\circ} \mathrm{C}$ and $200^{\circ} \mathrm{C}$ for $1 \mathrm{~h}$.

\subsection{Characterization}

The introduction of chemical bonds and functional groups onto the graphene surface was confirmed by X-ray photoelectron microscopy (XPS, AXIS-NOVA, Kratos Inc., USA) and Raman spectroscopy (Lab RAM HR, Horiba, Japan).The barrier properties of the bare and coated PI films were also examined. The water-vapor transmission rat of bare-PI and coated-PI film was measured using a water vapor transmission analyzer (AQUATRAN Model 1, Mocon, USA). The oxygen transmission rate of the bare and coated PIs at $25^{\circ} \mathrm{C}$ was measured using a gas transmission-rate tester (Toyoseiki, Japan). A flow of oxygen at $100 \mathrm{KPa}$ was fed to one side of a $2.5 \mathrm{~cm}$ diameter film sample. The fractured cross-section was observed for analysis using scanning electron microscopy (NOVA NanoSEM, FEI, USA).

\section{Results and Discussion}

Amino-phenyl groups are formed on the surface of GO through the diazonium-salt reaction, which was confirmed by the XPS analysis. Fig. 2a shows the spectra of the XPS C1s original and fitted curves of GO. GO had four major peaks, with positions at $284.6,286.6,288.0$ and $289.5 \mathrm{eV}$; which are attributed to the $\mathrm{C}=\mathrm{C}, \mathrm{C}-\mathrm{O}, \mathrm{C}=\mathrm{O}$ and $\mathrm{O}-\mathrm{C}=\mathrm{O}$ species, respectively; which indicate that the oxidation of graphite intended by using Hummer's method, occurred successfully. In the case of APGO, a new peak at $285.7 \mathrm{eV}$ was observed, which is the $\mathrm{C} 1 \mathrm{~s}$ attributed to a $\mathrm{C}-\mathrm{N}$ bond [17]. This result demonstrates the formation of covalent bonds between amino-phenyl groups and GO. N1s also showed three peaks at 397.7, 399.2, and $405.2 \mathrm{eV}$, corresponding to C-N, $-\mathrm{NH}_{2}$, and $\mathrm{Ar}-\mathrm{NH}_{2}$, respectively [18]. The peak intensity of the $-\mathrm{NH}_{2}$ groups of APGO (Fig. 2d) is higher than that of NPGO (Fig. $2 \mathrm{c}$ ), which reveals the reduction from $-\mathrm{NO}_{2}$ of NPGO to $\mathrm{NH}_{2}$. This 

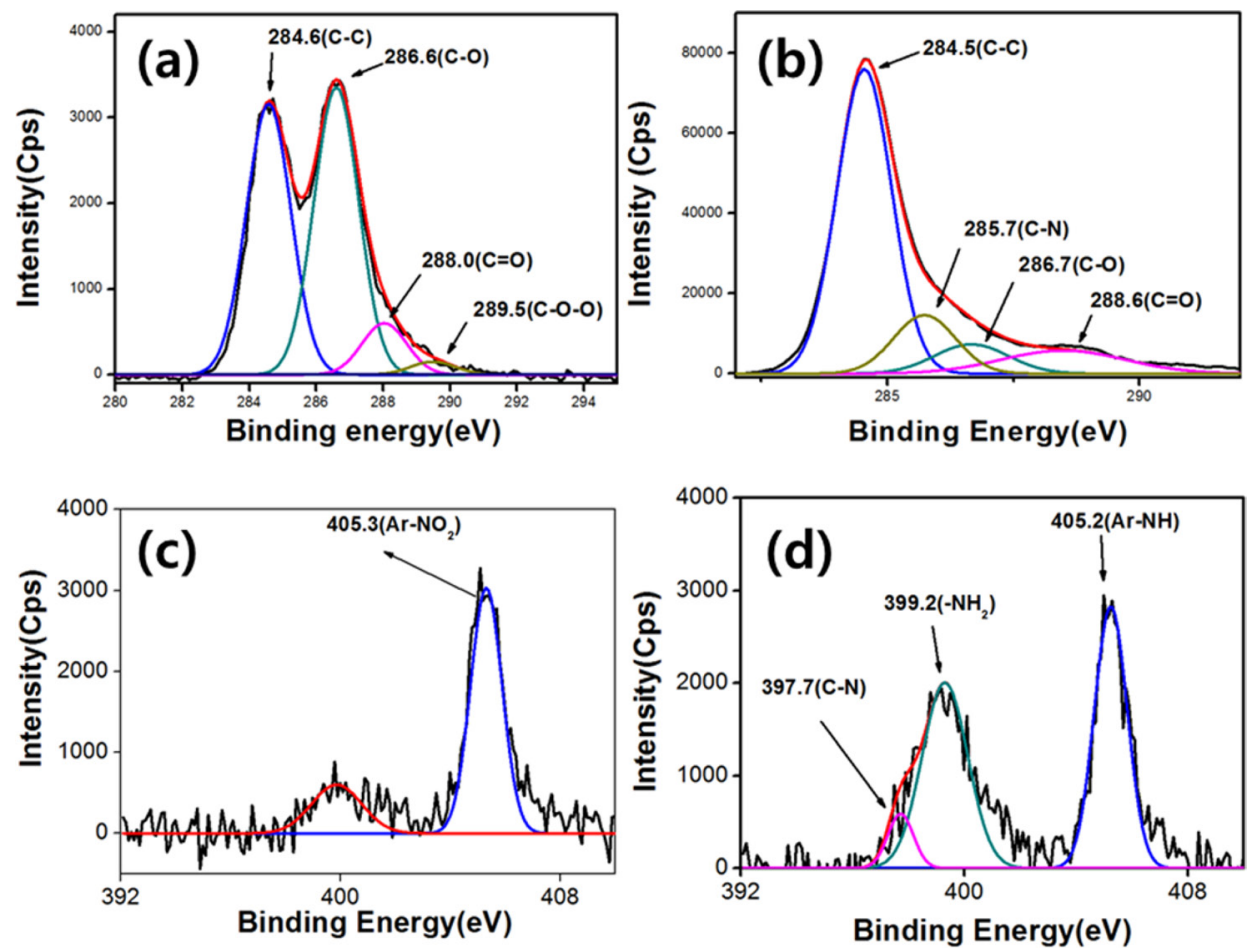

Fig. 2. X-ray photoelectron microscopy C1s spectra of GO (a), APGO (b), N1s spectra of NPGO (c) and APGO (d). GO: graphene oxide, APGO: amino-phenyl functionalized GO, NPGO: nitro-phenyl functionalized GO.

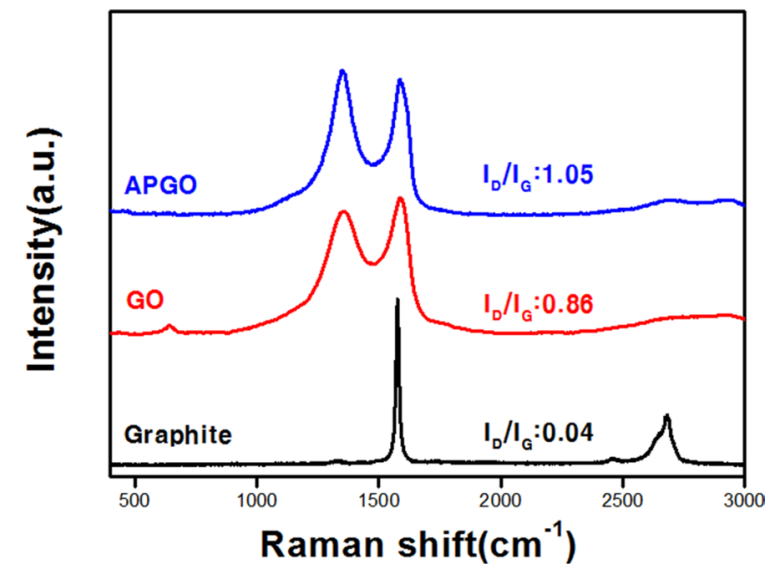

Fig. 3. Raman spectra and $\mathrm{I}_{\mathrm{D}} / \mathrm{I}_{\mathrm{G}}$ ratio of graphite, graphene oxide $(\mathrm{GO})$ and amino-phenyl functionalized GO (APGO).

clearly confirms the presence of $\mathrm{Ar}-\mathrm{NH}_{2}$ groups on the surface of graphene via the diazonium-salt reaction.

Fig. 3 shows the Raman spectra of Gr, GO and APGO. The Raman spectrum of APGO displays two strong peaks at 1580 and at $1350 \mathrm{~cm}^{-1}$, corresponding to the D and G-bands, respectively. The G-band is attributed to the first order scattering of the $\mathrm{E}_{2 \mathrm{~g}}$ phonon of the $s p^{2}$ carbon-carbon bond; while the D-band represents the defect sites associated with vacancies and grain boundaries $[19,20]$. For the diazonium-salt reaction, the $\mathrm{sp}^{2}$ of a carbon bond is converted to the $s p^{3}$-hybridized carbon atom. Therefore, the intensity ratio of $\mathrm{I}_{\mathrm{D}} / \mathrm{I}_{\mathrm{G}}$ provides direct evidence of the degree of functionalization $[21,22]$.

\section{Table 1. Barrier properties of pure $\mathrm{PI}$ and $\mathrm{rGO} / \mathrm{rAPGO} / \mathrm{PI}$}

\begin{tabular}{ccc} 
Sample & $\begin{array}{c}\text { Water vapor transmission } \\
\text { rate }\left(\mathrm{g} / \mathrm{m}^{2} \cdot \text { day }\right)\end{array}$ & $\begin{array}{c}\text { Oxygen transmission } \\
\text { rate }\left(\mathrm{cm}^{3} / \mathrm{m}^{2} \cdot a t m\right)\end{array}$ \\
\hline Pure PI & 58 & 213 \\
$\mathrm{rGO} / \mathrm{rAPGO} / \mathrm{PI}$ & 179 & 78 \\
\hline
\end{tabular}

PI: polyimide, rGO: reduced graphene oxide, rAPGO: reduced aminophenyl functionalized GO.

The ratio of $\mathrm{I}_{\mathrm{D}} / \mathrm{I}_{\mathrm{G}}$ of APGO was increased from 0.86 to 1.05 after amino-phenyl functionalization. This result also clearly indicates that the amino-phenyl functional group was covalently bonded onto the surface of the GO after surface functionalization.

Combining graphene with the polymer matrix made it possible to produce barrier polymer-composite films. The 2-dimensional structure of the graphene can improve the barrier properties of the polymer matrix. Herein, we investigated the water vapor transmission rate (WVTR) and oxygen transmission rate (OTR), of the prepared (rGO/rAPGO) $)_{10}$ deposited PI film (rGO/rAPGO/PI) and of the pure PI film. The WVTR and OTR of these samples are summarized in Table 1. In the case of WVTR, the transmission rate of the $\mathrm{rGO} /$ rAPGO/PI-composite film is higher than that of pure PI film. The WVTR depends mainly on the hydrophilic or hydrophobic characteristics of materials. The oxygen groups of GO and APGO cannot be completely removed during the imidization process. The remaining oxygen groups can still make membranes hydrophilic, compared to that of pure PI. Therefore, the WVTR of $\mathrm{rGO} / \mathrm{rAPGO} / \mathrm{PI}$ is higher than that of PI. However, OTR is strongly dependent on the barrier effect of the fillers. The OTR of the rGO/rAPGO/PI composite showed about $60 \%$ decrease compared that of pure PI. The large as- 
pect-ratio of graphene multilayers can maximize the diffusion length of gaseous molecules through the composites; in what is called a "tortuous path" $[23,24]$. Therefore, PI composites with rGO and rAPGO should exhibit lower OTR values than that of pure PI due to the barrier effect of the graphene film on the PI surface.

\section{Conclusions}

PI-graphene composite films were produced using a LBL assembly technique. The water-vapor barrier property of the composite membranes was not enhanced due to the hydrophilic nature of the graphene layers; while the oxygen barrier property was improved by $60 \%$ compared to pure PI membranes. This result indicates that water vapor transmission rate is strongly affected by the hydrophilic quality of the materials, and that the oxygen barrier property is affected by the barrier effect of fillers.

\section{Acknowledgements}

This work was supported by a grant from the Korea Institute of Science and Technology (KIST) Institutional Program, and by the Converging Research Center Program through the Ministry of Science, ICT \& Future Planning.

\section{References}

[1] Nair RR, Blake P, Grigorenko AN, Novoselov KS, Booth TJ, Stauber T, Peres NMR, Geim AK. Fine structure constant defines visual transparency of graphene. Science, 320, 1308 (2008). http://dx.doi. org/10.1126/science.1156965.

[2] Pham VH, Cuong TV, Nguyen-Phan T-D, Pham HD, Kim EJ, Hur SH, Shin EW, Kim S, Chung JS. One-step synthesis of superior dispersion of chemically converted graphene in organic solvents. Chem Commun, 46, 4375 (2010). http://dx.doi.org/10.1039/C0CC00363H.

[3] Huang W, Ouyang X, Lee LJ. High-performance nanopapers based on benzenesulfonic functionalized graphenes. ACS Nano, 6, 10178 (2012). http://dx.doi.org/10.1021/nn303917p

[4] Huang P, Zhu H, Jing L, Zhao Y, Gao X. Graphene covalently binding aryl groups: conductivity increases rather than decreases. ACS Nano, 5, 7945 (2011). http://dx.doi.org/10.1021/nn2023232.

[5] Huang X, Qi X, Boey F, Zhang H. Graphene-based composites. Chem Soc Rev, 41, 666 (2012). http://dx.doi.org/10.1039/C1CS15078B.

[6] Chae BJ, Kim DH, Jeong IS, Hahn JR, Ku BC. Electrical and thermal properties of poly(p-phenylene sulfide) reduced graphite oxide nanocomposites. Carbon Lett, 13, 221 (2012). http://dx.doi. org/10.5714/CL.2012.13.4.221.

[7] Koerner H, Price G, Pearce NA, Alexander M, Vaia RA. Remotely actuated polymer nanocomposites-stress-recovery of carbonnanotube-filled thermoplastic elastomers. Nat Mater, 3, 115 (2004). http://dx.doi.org/10.1038/nmat1059.

[8] Kim H, Miura Y, Macosko CW. Graphene/polyurethane nanocomposites for improved gas barrier and electrical conductivity. Chem Mater, 22, 3441 (2010). http://dx.doi.org/10.1021/cm100477v.

[9] Huang HD, Ren PG, Chen J, Zhang WQ, Ji X, Li ZM. High barrier graphene oxide nanosheet/poly(vinyl alcohol) nanocom- posite films. J Membr Sci, 409-410, 156 (2012). http://dx.doi. org/10.1016/j.memsci.2012.03.051.

[10] Ku BC, Kumar J, Blumstein A, Kim DW, Samuelson LA. Barrier properties of ordered multilayer polymer nanocomposites. In Schwarz JA, Contescu CI, Putyera K, eds. Dekker Encyclopedia of Nanoscience and Nanotechnology, Marcel Dekker, New York, 213 (2004). http://dx.doi.org/10.1201/9781439834398.ch9.

[11] Decher G. Fuzzy Nanoassemblies: toward layered polymeric multicomposites. Science, 277, 1232 (1997). http://dx.doi.org/10.1126/ science.277.5330.1232.

[12] Yang YH, Bolling L, Priolo MA, Grunlan JC. Super gas barrier and selectivity of graphene oxide-polymer multilayer thin films. Adv Mater, 25, 503 (2013). http://dx.doi.org/10.1002/adma.201202951.

[13] Qu Q, Gu C, Gu Z, Shen Y, Wang C, Hu X. Layer-by-layer assembly of polyelectrolyte and graphene oxide for open-tubular capillary electrochromatography. J Chromatogr A, 1282, 95 (2013) http://dx.doi.org/10.1016/j.chroma.2013.01.055.

[14] Park JS, Cho SM, Kim WJ, Park J, Yoo PJ. Fabrication of graphene thin films based on layer-by-layer self-assembly of functionalized graphene nanosheets. ACS Appl Mater Interfaces, 3, 360 (2011). http://dx.doi.org/10.1021/am100977p.

[15] Lee DW, Hong TK, Kang D, Lee J, Heo M, Kim JY, Kim B-S, Shin HS. Highly controllable transparent and conducting thin films using layer-by-layer assembly of oppositely charged reduced graphene oxides. J Mater Chem, 21, 3438 (2011). http://dx.doi org/10.1039/C0JM02270E.

[16] Hummers WS, Jr., Offeman RE. Preparation of graphitic oxide. J Am Chem Soc, 80, 1339 (1958). http://dx.doi.org/10.1021/ ja01539a017.

[17] Shang J, Ma L, Li J, Ai W, Yu T, Gurzadyan GG. The origin of fluorescence from graphene oxide. Sci Rep, 2, 792 (2012). http:// dx.doi.org/10.1038/srep00792.

[18] Park OK, Hwang JY, Goh M, Lee JH, Ku BC, You NH. Mechanically strong and multifunctional polyimide nanocomposites using amimophenyl functionalized graphene nanosheets. Macromolecules, 46, 3505 (2013). http://dx.doi.org/10.1021/ma400185j

[19] Kim NH, Kuila T, Lee JH. Simultaneous reduction, functionalization and stitching of graphene oxide with ethylenediamine for composites application. J Mater Chem A, 1, 1349 (2013). http://dx.doi. org/10.1039/C2TA00853J.

[20] Park OK, Hahm MG, Lee S, Joh HI, Na SI, Vajtai R, Lee JH, Ku BC, Ajayan PM. In situ synthesis of thermochemically reduced graphene oxide conducting nanocomposites. Nano Lett, 12, 1789 (2012). http://dx.doi.org/10.1021/nl203803d.

[21] Ferrari AC, Meyer JC, Scardaci V, Casiraghi C, Lazzeri M, Mauri F, Piscanec S, Jiang D, Novoselov KS, Roth S, Geim AK. Raman spectrum of graphene and graphene layers. Phys Rev Lett, 97, 187401 (2006). http://dx.doi.org/10.1103/PhysRevLett.97.187401.

[22] Park OK, Lee S, Joh HI, Kim JK, Kang PH, Lee JH, Ku BC. Effect of functional groups of carbon nanotubes on the cyclization mechanism of polyacrylonitrile (PAN). Polymer, 53, 2168 (2012). http://dx.doi.org/10.1016/j.polymer.2012.03.031.

[23] Priolo MA, Gamboa D, Holder KM, Grunlan JC. Super gas barrier of transparent polymer-clay multilayer ultrathin films. Nano Lett, 10, 4970 (2010). http://dx.doi.org/10.1021/n1103047k

[24] Tseng IH, Liao YF, Chiang JC, Tsai MH. Transparent polyimide/ graphene oxide nanocomposite with improved moisture barrier property. Mater Chem Phys, 136, 247 (2012). http://dx.doi. org/10.1016/j.matchemphys.2012.06.061 